\title{
The association between serum prolactin levels and interleukin- 6 and systemic lupus erythematosus activity
}

\author{
W.A. Wan Asyraf, M.S. Mohd Shahrir, W. Asrul, A.W. Norasyikin, \\ O. Hanita, W.Y. Kong, M.T. Azmi \\ University Kebangsaan Malaysia Medical Centre, Cheras, Kuala Lumpur, Malaysia
}

\begin{abstract}
SUMMARY
Based on the recent evidence of association between hyperprolactinemia and systemic lupus erythematosus disease activity (SLEDAI), a study was conducted to analyze the association of hyperprolactinemia with lupus nephritis disease activity.

In this cross-sectional study, the analysis was conducted on SLE patients who visited the University Kebangsaan Malaysia Medical Centre (UKMMC) Nephrology Clinic from August 2015 till February 2016. The disease activity was measured using the SLEDAI score, with more than 4 indicating active lupus nephritis.

Basal resting prolactin level was analyzed in 43 patients with lupus nephritis, in $27.9 \%$ of them had raised serum prolactin. The median of serum prolactin level at 0 minutes was $19.91 \mathrm{ng} / \mathrm{mL}$ (IQR: 15.95-22.65 ng/ $\mathrm{mL}$ ) for active lupus nephritis, which was significantly higher compared to the median of serum prolactin level of $14.34 \mathrm{ng} / \mathrm{mL}$ (IQR: $11.09-18.70 \mathrm{ng} / \mathrm{mL}$ ) for patients in remission ( $\mathrm{p}=0.014)$. The serum prolactin level positively correlated with SLEDAI ( $\left.\mathrm{rho}_{\mathrm{s}}: 0.449, \mathrm{p}=0.003\right)$ and the UPCI level in lupus nephritis patients $\left(\mathrm{rho}_{\mathrm{s}}\right.$ : $0.241, \mathrm{p}=0.032$ ). The results were reproduced when the serum prolactin was repeated after 30 minutes. However, the serum prolactin levels at 0 minutes were higher than those taken after 30 minutes $(p=0.001)$. An assessment of serum IL-6 levels found that the active lupus nephritis patients had a higher median level of $65.91 \mathrm{pg} /$ $\mathrm{mL}$ (IQR: $21.96-146.14 \mathrm{pg} / \mathrm{mL}$ ) compared to the in-remission level of $15.84 \mathrm{pg} / \mathrm{mL}$ (IQR: $8.38-92.84 \mathrm{pg} / \mathrm{mL}$ ), $(\mathrm{p}=0.039)$. Further correlation analysis revealed that there was no statistical correlation between the interleukin (IL)-6 levels with serum prolactin, SLEDAI and other lupus nephritis parameters. An ROC curve analysis of serum prolactin at 0 minutes and serum prolactin after 30 minutes and IL-6 levels for prediction of SLE disease activity provided the cutoff value of serum prolactin at 0 minutes, which was $14.63 \mathrm{ng} / \mathrm{mL}$ with a sensitivity of $91.7 \%$ and specificity of $58.1 \%$ and AUC of 0.74 ( $\mathrm{p}=0.015)$.

This study concurred with the previous findings that stated that hyperprolactinemia is prevalent in SLE patients and correlated with clinical disease activity and UPCI level. The baseline of the fasting serum prolactin level was found to be a sensitive biomarker for the evaluation of lupus nephritis disease activity.
\end{abstract}

Key words: Prolactin; Interleukin-6; Systemic lupus erythematosus.
Conference presentation The Abstract was presented in the Poster Session of the $12^{\text {th }}$ International Congress on SLE \&, $7^{\text {th }}$ Asian Congress on Autoimmunity, and is available at: https://lupus.bmj.com/ content/4/Suppl_1/A135.1

\section{INTRODUCTION}

ystemic lupus erythematosus (SLE) is an autoimmune disease that predominantly affects women in their reproductive age, with the female to male ratio of $8.6: 1$ $(1,2)$. The abnormalities of the immune response in SLE patients are contributed by the interplay of multiple factors, including genetics, environment, and hormones (3). Prolactin is a polypeptide hormone produced by the anterior pituitary gland and found to be associated with the immune regulation in SLE. Several studies have been conducted since the 1980s to understand the association of this lactogenic hormone with the development of autoimmune diseases and it was discovered to have been produced by other extra-pituitary organs and cells, including those from the immune system. It also accelerated the breakdown of immune tolerance by promoting the survival of $B$ and $T$ cells, dendritic cells, as well as macrophages (4). The role of prolactin as an immune-modulator was prov-
Corresponding author: Mohamed Said Mohd Shahrir Rheumatology and Internal Medicine Department, University Kebangsaan Malaysia Medical Centre, Cheras, Kuala Lumpur, Malaysia E-mail: drobiwan@gmail.com 
en by further studies that found structural similarity among the members of cytokines such as interleukin (IL)-2, IL-7, GM-CSF, and erythropoietin. Its receptor configuration also had a similar molecular structure to that of the cytokine/hematopoietic receptor family $(5,6)$.

The prevalence of hyperprolactinemia (HPRL) in a normal healthy population is lower than $5 \%$. From previous studies, the prevalence of mild to moderate HPRL varied between 20-30\% in SLE patients (7). Although previous attempts to associate HPRL in SLE with disease activity produced contradictory findings, several other studies have proved that HPRL found in SLE patients was associated with disease activity and specific organ involvement. Central nervous system, cutaneous, as well as articular involvement and kidney with serological disease activity correlated with HPRL in SLE patients (8-10). These findings further strengthened the association of prolactin in the pathogenesis of SLE. Due to the conflicting findings and lack of data in this area, a study was thereby conducted to examine the association of hyperprolactinemia with lupus nephritis disease activity (SLEDAI) and the IL-6 level in a specific subset of SLE patients with lupus nephritis.

\section{OBJECTIVES}

\section{AND ETHICS APPROVAL}

The primary objective was to determine the correlation between high prolactin level and lupus nephritis disease activity in the University Kebangsaan Malaysia Medical Centre (UKMMC).

Secondary objectives were: i) to determine the correlation between high prolactin level and the IL-6 level in lupus nephritis patients in UKMMC; ii) to determine the correlation between the IL-6 levels and lupus nephritis disease activity in UKMMC.

\section{Research questions and study hypotheses}

The study aimed to identify explanations on the following issues:

i) Does high prolactin level correlate with lupus nephritis disease activity? ii) Does a high prolactin level correlate with IL-6 level in lupus nephritis patients?

iii) Does high IL-6 level correlate with lupus nephritis disease activity?

\section{Ethics approval}

This study acquired the ethical approval from the Research Ethics Committee of UKM Medical Centre (Code No: FF-2015280). All protocols used were approved. Patients were required to sign an informed consent form and reserved the right to withdraw from the study.

\section{METHODOLOGY} AND STUDY DESIGN

\section{Study design}

This was a cross-sectional study. It was carried out at the UKMMC and involved patients followed up under the Nephrology and Rheumatology Clinic at the UKMMC from August 2015 till February 2016.

All patients who met the inclusion criteria, and were without those for exclusion, criteria were enrolled in the study after acquiring ethics approval (Code: FF2015-280). The patient was considered involved in the study after he or she agreed and signed the written informed consent.

\section{Patient population}

All patients with clinical and biopsy-proven lupus nephritis in UKMMC met the following inclusion and non-exclusion criteria:

Inclusion criteria:

1) patients with a diagnosis of SLE with lupus nephritis based on the SLICC criteria may be recruited;

2) age $\geq 18$ years old till 52 years old for female (average age of menopause) and age $\geq 18$ years old for male;

$3)$ those willing to give informed consent.

\section{Exclusion criteria:}

a) patients who do not give informed consent;

b) presence of a specific cause, which may result in the increase of prolactin:

1. endocrinopathy: pituitary adenoma, hypothyroidism; 
2. drugs: antipsychotics that inhibit dopamine (haloperidol, phenothiazine, clozapine, olanzapine, etc.), antiemetic (metoclopramide, domperidone), and hormones (estrogen and androgen);

3. raised creatinine $>175 \mathrm{mmol} / \mathrm{L}$ chronic kidney disease may impair the excretion of prolactin;

4. any derangement in ALT, AST, and bilirubin;

5. pregnancy and nursing mother; and vi) obvious infections: management as per sepsis.

Factors that may decrease the prolactin level:

1) menopause;

2) dopamine agonist (bromocriptine and L-dopa).

The sample size was calculated using the Power and Sample Size (PS) 2 Version 3 software (2011), whereby the outcome was dichotomous. The precision $(\alpha) 0.05$ power was at $80 \%$, with the assumption that a possible $10 \%$ would drop out. The prevalence of lupus nephritis reference according to Jacobi et al. was $76 \%$ with hyperprolactinemia and $42 \%$ at a normal prolactin level. Therefore, the minimum sample size needed (11) was 93 (HPRL group: 26 and non-HPRL group: 67).

\section{Data collection}

After fulfilling the inclusion and exclusion criteria, all patients with informed consent were interviewed by the researcher. The demographic data were recorded, as shown in the Patient's Data Collection.

Fasting and resting blood samples were taken for full blood count, renal profile, liver function test, thyroid function test, antinuclear antibody, double-stranded DNA, IL-6, and complement levels. Urine samples were also collected for examination, microscopic analysis, and urine protein creatinine index.

The patients' medications and baseline comorbidities were recorded.

\section{Materials for assessment of disease activity}

SLE patients with lupus nephritis disease activity were assessed using SLEDAI 2000 and any level >4 was considered active.

\section{Measurement of serum prolactin}

Each patient's venous blood samples were taken between 9:00 a.m. - 12:00 p.m. under fasting and resting conditions without hormonal or drug stimulus. They were taken at 0 minutes and after 30 minutes with minimal trauma.

The blood samples were centrifuged and the serum was aliquoted and stored at $-70^{\circ} \mathrm{C}$ until analysis. The serum prolactin was determined by the ARCHITECT Prolactin assay, which is a two-step immunoassay that determines the presence of prolactin in human serum and plasma using the chemiluminescent microparticle immunoassay technology with flexible assay protocols, or otherwise referred to as Chemiflex. The assay used $\mathrm{ng} / \mathrm{mL}$ as the default result unit with a conversion factor of 21 for an alternate result unit of mIU/L. The intra-assay and inter-assay coefficient of variations were $3.3-4.7 \%$ and $2.3-3.8 \%$ respectively. Patients were regarded as hyperprolactinemic with a serum prolactin concentration of $>16 \mathrm{ng} / \mathrm{mL}$ for males and $>20 \mathrm{ng} / \mathrm{mL}$ for females.

\section{Measurement of autoantibodies}

QUANTA Lite ${ }^{\circledR}$ dsDNA enzyme-linked immunosorbent assay (ELISA) by Inova Diagnostics was used to detect IgG-antidsDNA with an ELISA for the semi-quantitative detection of anti-dsDNA autoantibodies in human serum. The samples were analyzed in the Immunology Laboratory at UKMMC.

\section{Measurement of interleukin-6 level}

The interleukin- 6 concentration was measured using a BD OptEIA ELISA kit that uses a monoclonal antibody, which is a specific test with a solid phase sandwich ELISA. The IL-6 in each patient's serum was determined from the standard curve plotted based on the pre-determined standard IL- 6 concentrations. The test was performed and analyzed in the Immunology Laboratory at UKMMC. 


\section{RESULTS}

This was a cross-sectional study, whereby 71 patients with systemic lupus erythematosus diagnosed with lupus nephritis were approached for recruitment into the study from August 2015 till February 2016. 10 patients refused to participate, while 11 of them were excluded (five were on medication, which might have altered the prolactin level; three had premature menopause and three were pregnant). We were able to recruit 50 patients, but only 43 samples could be analyzed, as seven samples were damaged.

\section{Demographic and baseline characteristics}

The study population comprised 42 females and one male. In general, the median age of this population was 33 years old (IQR: $30-$ 36 years). It consisted, furthermore, of 21 Chinese (48.8\%), 20 Malays (46.5\%), and 2 others $(4.7 \%)$. The median duration of the SLE diagnosis was 8 years (IQR: 6-13 years).

Based on SLEDAI-2000, only 12 patients $(27.9 \%)$ were active and 31 patients $(72.1 \%)$ in remission. The median age of patients with active lupus nephritis was 30.5 years (IQR: 29.3-36.0 years), while the median age of patients in remission was 34.0 years (IQR: 31.0-37.0 years).

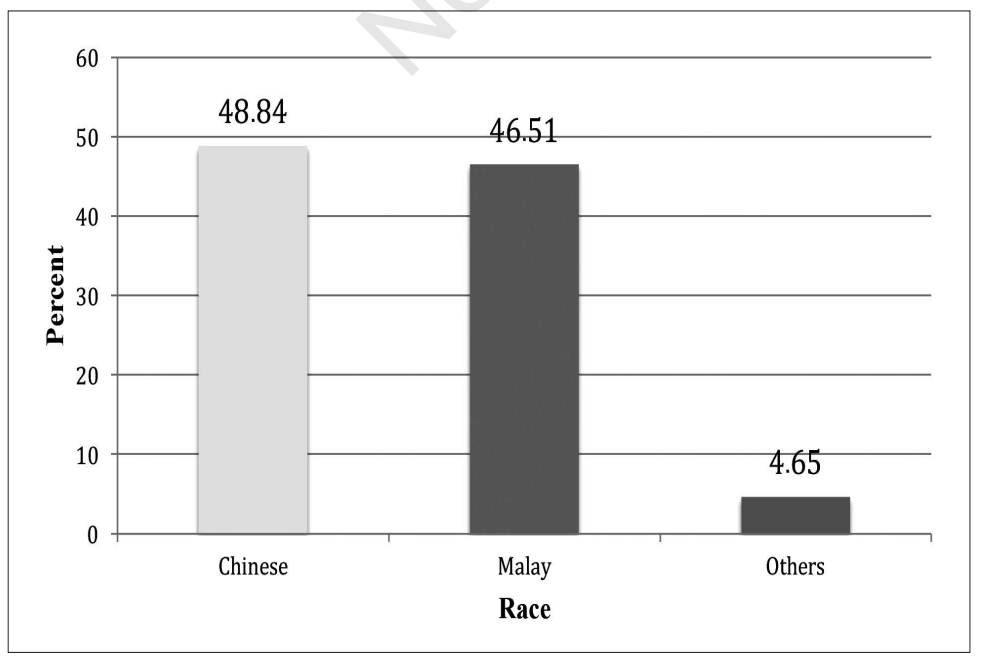

Figure 1 - Distribution of lupus nephritis patients according to race in University Kebangsaan Malaysia Medical Centre.
The median duration form diagnosis for active lupus nephritis was 7.5 years (IQR: 4.3-12.5 years), while for lupus nephritis in remission it was 8 years (IQR: 7.0-13.0 years). The results of the urine protein creatinine index showed a median of $0.08 \mathrm{~g} /$ mmol creatinine (IQR: 0.05-0.24 g/mmol) in active lupus nephritis patients, but it was lower at $0.02 \mathrm{~g} / \mathrm{mmol}$ creatinine (IQR: 0.01-0.03 g/mmol) for lupus nephritis patients in remission (Figure 1).

\section{Prolactin and disease activity}

The prevalence of high prolactin is $27.9 \%$ in this population. Out of the 12 patients with high prolactin, six $(50 \%)$ of had high prolactin and inactive disease. However, in the subgroup of patients in remission, the majority of the patients had a normal prolactin level $\mathrm{OH} 25$ (80.6\%). Only six (19.4\%) patients had a high prolactin level. When the median prolactin level at 0 minutes in active lupus nephritis patients compared with the median prolactin level in lupus nephritis patients in remission was analyzed, it was discovered to be statistically significantly higher in the active lupus nephritis subgroup ( $\mathrm{p}=0.014)$. Similarly, a statistically significant higher median value was found in the active lupus nephritis compared to the remission subgroup for the prolactin level at 30 minutes $(\mathrm{p}=0.009)$, as shown in Table I and Figure 2.

There was also a positive correlation between prolactin level and disease activity score (SLEDAI-2000) based on the Spearman correlation coefficient. It showed rho: $0.449, \mathrm{p}=0.003$ for 0 -minute prolactin and $\mathrm{rho}_{\mathrm{s}}: 0.424, \mathrm{p}=0.005$ for the 30 -minute prolactin level. These are depicted in Figures 3 and 4.The Chi-square analysis showed a significant association between prolactin level and lupus nephritis disease activity with $\mathrm{p}=0.044$. Based on the Wilcoxon signed rank test, there were significant differences between serum prolactin levels taken at 0 minutes compared to the serum prolactin levels at 30 minutes with $\mathrm{p}=0.001$. This showed a higher level of serum prolactin at 0 minutes compared to the 30-minute level. 
Table I - Comparison of lupus nephritis patients in active and remission status in UKMMC.

\begin{tabular}{|l|c|c|c|}
\hline & Active & Remission & p-value \\
\hline Age, median (IQR) & $30.5(29.3-36.0)$ & $34.0(31.0-37.0)$ & 0.211 \\
\hline Years of diagnosis, median (IQR) & $7.5(4.3-12.5)$ & $8.0(7.0-13.0)$ & 0.446 \\
\hline Creatinine, mmol/l, median (IQR) & $70.35(57.65-81.65)$ & $64.80(60.20-71.80)$ & 0.414 \\
\hline SLEDAl, median (IQR) & $8(6-12)$ & $2(0-4)$ & 0.005 \\
\hline UPCl, g/mmol creatinine, median (IQR) & $0.08(0.05-0.24)$ & $0.02(0.01-0.03)$ & 0.001 \\
\hline Prolactin level, ng/mL 0 min, median (IQR) & $19.91(15.95-22.65)$ & $14.34(11.09-18.70)$ & 0.014 \\
\hline Prolactin level, $\mathrm{ng} / \mathrm{mL} 30$ min, median (IQR) & $17.26(15.53-21.22)$ & $12.64(10.01-17.30)$ & 0.009 \\
\hline IL-6 level, pg/mL, median (IQR) & $65.91(21.96-146.14)$ & $15.84(8.38-92.84)$ & 0.039 \\
\hline Prednisolone dose, mg, median (IQR) & $17.5(10.0-30.0)$ & $10.0(5.0-10.0)$ & 0.001 \\
\hline Prolactin scale high, $\mathrm{n}$ (\%) & $6(50 \%)$ & $6(19.4 \%)$ & 0.044 \\
\hline Prolactin scale normal, $\mathrm{n}(\%)$ & $6(50)$ & $25(80.6)$ & 0.044 \\
\hline Total cases, $\mathrm{n}$ (27) & $12(27.9)$ & $31(72.1)$ & \\
\hline
\end{tabular}

In addition to the above results, the serum prolactin level at 0 minutes (rho: 0.241 , $\mathrm{p}=0.032$ ) and after 30 minutes (rho: 0.314 , $\mathrm{p}=0.040$ ) showed positive correlation with the UPCI level in lupus nephritis patients. Although there was no statistically significant association between the serum prolactin level and intensity of the therapy (prednisolone more than $10 \mathrm{mg}$ or less), ( $p=0.251$ based on Fisher's exact test), the median prednisolone dose in active lupus nephritis patients was statistically significantly higher compared to the remission subgroup as shown in Table I. Further analysis showed no significant correlation between the serum prolactin with other parameters, including white blood cell count, hemoglobin count, platelet count, lymphocyte count, C3 and C4 levels, as shown in Table II. Based on the Chi-square analysis, no statistically significant association was found between the serum prolactin and the anti-dsDNA and ANA levels ( $\mathrm{p}=0.438$ and $\mathrm{p}=0.836$ ).

Based on the receiver operating characteristic (ROC) curve of serum prolactin at 0 minutes and serum prolactin after $30 \mathrm{~min}$ utes, as well as the IL- 6 level for the prediction of SLE diseases activity, the cutoff value of serum prolactin at 0 minutes was $14.63 \mathrm{ng} / \mathrm{mL}$ versus $15.03 \mathrm{ng} / \mathrm{mL}$ of serum prolactin at 30 minutes, with a sensitivity of $91.7 \%$ versus $83.3 \%$, a specificity of $58.1 \%$ versus $71.0 \%$, and an AUC

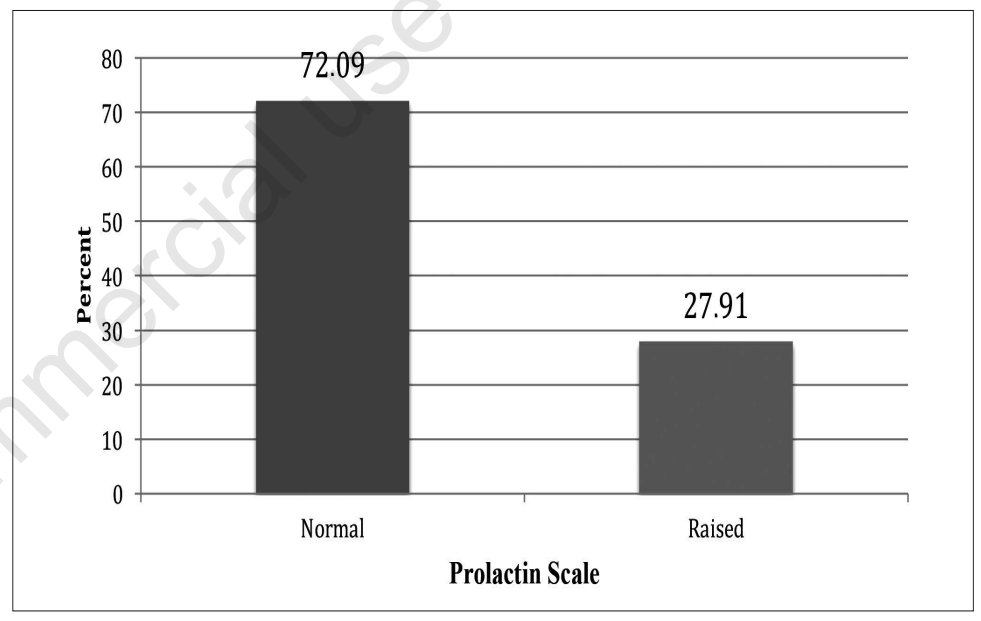

Figure 2 - Prevalence of raised and normal serum prolactin in lupus nephritis patients in University Kebangsaan Malaysia Medical Centre.

Table II - Correlation between prolactin level and other parameters.

\begin{tabular}{|l|c|c|}
\hline Parameter & Rhos & p-value \\
\hline SLEDAl-2000 & $0.449_{0}$ & 0.003 \\
\hline & $0.424_{30}$ & 0.005 \\
\hline UPCl & $0.241_{0}$ & 0.032 \\
& $0.314_{30}$ & 0.040 \\
\hline WBC & 0.174 & 0.263 \\
\hline Hemoglobin count & -0.087 & 0.590 \\
\hline Platelet & -0.254 & 0.425 \\
\hline Lymphocyte count & -0.060 & 0.704 \\
\hline C3 & 0.250 & 0.105 \\
\hline C4 & 0.081 & 0.607 \\
\hline
\end{tabular}

Rho*, ${ }^{*}={ }_{0}$ or ${ }_{30}$ for prolactin 0 or 30 minutes. 
of 0.74 versus 0.76 (95\% CI of $0.58-0.90$ versus 0.60-0.91).

The area under the curve for IL-6 was poor at $0.47(\mathrm{p}=0.787)$. In addition, the ROC curve analysis of prolactin at 0 minutes and prolactin at 30 minutes proved that both were sensitive as biomarkers of disease activity, with similar good PPV of 91.7 and low NPV of 58.1.

\section{Interleukin-6 association with serum} prolactin, SLEDAI, and other parameters To fulfill the study's secondary objectives, we further analyzed and found a statistically significant $(p=0.039)$ higher level of serum IL-6 in active lupus nephritis patients compared to the remission subgroup.

However, the serum IL-6 level did not correlate with the serum prolactin levels at 0 and 30 minutes $\left(\right.$ rho $_{\mathrm{s}}: 0.282, \mathrm{p}=0.106$, and rho $_{\mathrm{s}}$ : $0.190, \mathrm{p}=0.283$ ) and lupus nephritis disease activity $\left(\mathrm{rho}_{\mathrm{s}}: 0.254, \mathrm{p}=0.147\right)$. In this study, the serum IL-6 levels had no significant statistical correlation with other parameters in SLE with lupus nephritis as summarized in Table III.

\section{DISCUSSION}

The serum prolactin was assessed at 0 minutes and 30 minutes under fasting and resting conditions, as its half-life is of 20-30 minutes (12). This was important, enabling the study to exclude the serum

Table III - Correlation between serum interleukin-6 level and other parameters.

\begin{tabular}{|l|c|c|}
\hline Parameter & Rhos & p-value \\
\hline Prolactin & $0.282_{0}$ & 0.106 \\
\hline & $0.190_{30}$ & 0.283 \\
\hline SLEDAI & 0.254 & 0.147 \\
\hline UPCI & 0.279 & 0.111 \\
\hline WBC & 0.064 & 0.721 \\
\hline Hemoglobin count & -0.074 & 0.678 \\
\hline Platelet & -0.107 & 0.559 \\
\hline Lymphocyte count & -0.049 & 0.763 \\
\hline C3 & 0.332 & 0.055 \\
\hline C4 & 0.252 & 0.151 \\
\hline
\end{tabular}

$\mathrm{RhO}^{*},{ }^{*}{ }_{0}$ or $_{30}$ for prolactin 0 or 30 minutes. prolactin pulse release effect rather than the continuous release effect from the anterior pituitary as a response towards a stress event. Other conditions or drugs that may lead to the increment of serum prolactin levels or inhibition of prolactin release were also excluded. A past study found a consistent increased level of serum prolactin in SLE and noted a declining serum prolactin level compared to the subsequent sampling (13). A similar finding was discovered in this study where the median value of the first samples at 0 minutes was significantly higher in comparison to the median value of second samples of serum prolactin $(\mathrm{p}=0.005)$. This could have been determined by stress and dysregulation due to the underlying autoimmune disease, SLE (13).

\section{Prolactin association with systemic lupus erythematosus and disease activity}

The findings in this study, which showed that hyperprolactinemia was prevalent in SLE disease and associated with SLE disease activity, or specifically lupus nephritis disease activity, were in line with previous studies $(9,14,15)$.

In 1997, Huang et al. studied 30 patients to identify the correlation between HPRL and SLEDAI and serological markers and found that a higher mean prolactin value at $19.35 \mathrm{ng} / \mathrm{dL}$ was noted in SLE patients compared to normal subjects, at $12.01 \mathrm{ng} /$ dL (16). A subsequent study by Jacobi et al., which studied 60 SLE patients, produced similar findings of higher mean prolactin level of $17.4 \mathrm{ng} / \mathrm{mL}$ in SLE patients compared to normal healthy subjects who had a lower mean value of 6.3 $\mathrm{ng} / \mathrm{mL}(\mathrm{p}=0.001)$. However, the prolactin level was found to be significantly correlated with SLE disease activity assessed using the European Consensus Lupus Activity Measurement (ECLAM), (rho $=0.54$, $\mathrm{p}<0.001)$. Similarly, a positive correlation was also found with anti-dsDNA level (14). Comparatively in this study, a higher median value of serum prolactin at 0 minutes in active disease of $19.91 \mathrm{ng} / \mathrm{mL}$ (IQR: 15.95$22.65 \mathrm{ng} / \mathrm{mL}$ ) was discovered compared to a median value of serum prolactin in lupus 


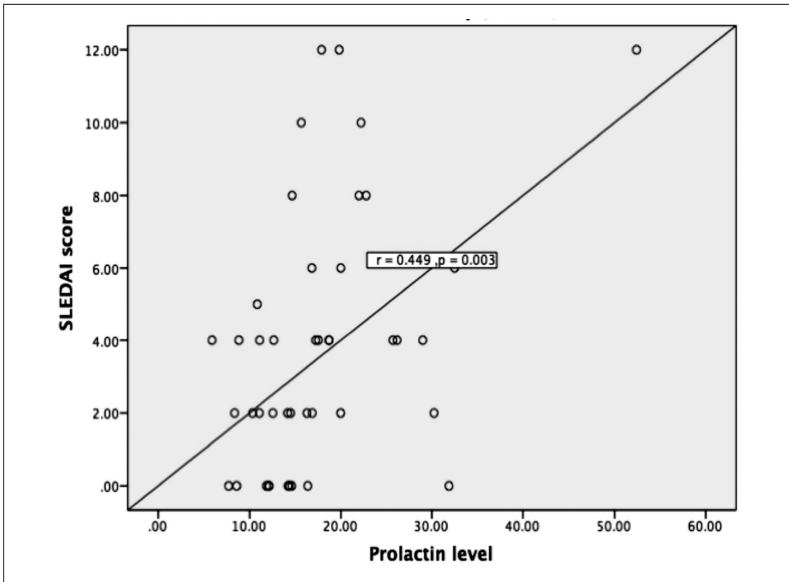

Figure 3 - Scattered diagram shows correlation between prolactin levels 0 minute and disease activity (SLEDAI).

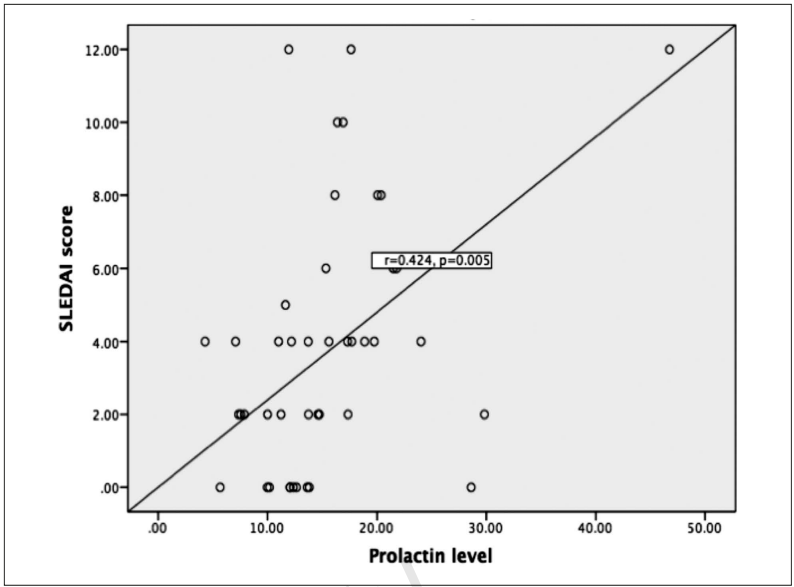

Figure 4 - Scattered diagram shows correlation between prolactin levels 30 minutes and disease activity (SLEDAI). nephritis in remission $(\mathrm{p}=0.014)$, while the median value of serum prolactin at $30 \mathrm{~min}$ utes in active lupus nephritis was also high at $17.26 \mathrm{ng} / \mathrm{mL}$ (IQR: $15.53-21.22 \mathrm{ng} / \mathrm{mL}$ ) and $\mathrm{p}=0.009$. The serum prolactin at the 0 -minute level was similar to the findings by Huang et al., while the serum prolactin at 30 minutes was in line with Jacobi et al.'s findings.

These two studies showed contradictory results, which can be explained by the different methods of analysis of the serum prolactin level and the SLE disease assessment scoring system applied. Another explanation for the negative correlation between the serum prolactin and disease activity in a few past studies was the presence of the anti-prolactin antibodies complex (macroprolactin), which is associated with lower disease activity (17). Normal daily variation of serum prolactin level throughout the day may also explain the variation of findings in a previous study (13). Therefore, this study suggested taking the serum prolactin level at fasting and resting conditions between 9:00 a.m. and 12:00 p.m.

Recently, Ugarte-Gil et al. assessed the association of serum prolactin level with damage accrual in SLE patients and found positive correlation between serum prolactin levels and the System Lupus International Collaborating Clinics/American College of Rheumatology (SLICC/ACR) damage index (SDI), $(\mathrm{rho}=0.29, \mathrm{p}<0.001)$.
After further analysis, a high prolactin level was discovered to be associated with renal, cardiac peripheral vascular, and premature gonadal failure domains of the SDI (18). This explained the findings of this study, which discovered a positive correlation between the serum prolactin and UPCI level in lupus nephritis patients $(\mathrm{p}=0.032$ and $\mathrm{p}=0.040$ ). Even so, no statistically significant associations between other serological tests (ANA and anti-dsDNA) with the serum prolactin level, noted by a few other studies, was found $(14,15)$.

As seen in Figure 5, the ROC curve analysis of serum prolactin at 0 and 30 minutes proved that serum prolactin can act as a

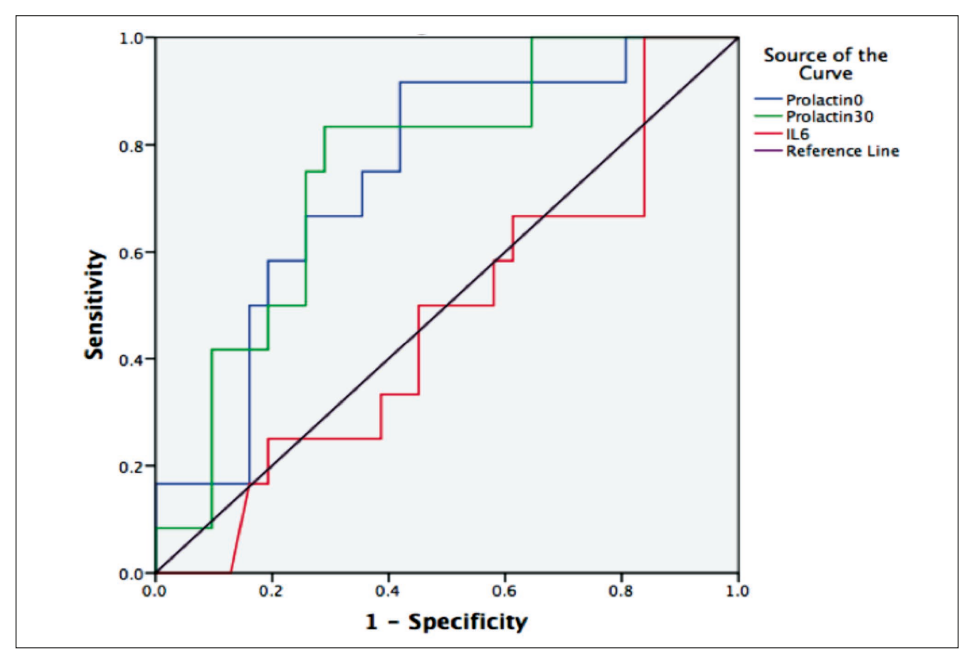

Figure 5 - Receiver operating characteristic curve. 
sensitive biomarker of disease activity. The sensitivity of the serum prolactin at 0 minutes was higher, at $97.1 \%$, while the sensitivity of serum prolactin at 30 minutes was $83.3 \%$. Positive predictive value (PPV) was high, at $91.7 \%$ for both serum prolactin timings.

\section{Serum prolactin, interleukin-6, and lupus nephritis}

Recently, Cai et al. studied 40 SLE patients and found that HPRL patients had a higher IL-6 level secreted by the peripheral blood mononuclear cells (PBMCs). The same study also found an increment in the IL-6 secretions from the PBMC after an in vitro stimulation by recombinant human prolactin $(p=0.001)(19,20)$. Furthermore, a study on single-nucleotide polymorphism explained the above mechanism through the discovery in African American and European American women with lupus and presence of -1149TT genotype of a correlation with higher levels of prolactin in serum and prolactin gene expression in peripheral blood mononuclear cells. Similarly, this study also found a higher level of serum IL-6 in lupus patients, especially in the African American subgroup (3).

In this study, the researchers found a higher median value of serum IL-6 in the active lupus nephritis subgroup, which was statistically significant $(\mathrm{p}=0.039)$. However, further correlation analysis revealed no meaningful correlation between the serum IL-6 level and SLEDAI and other clinical parameters. The findings were similar with Ripley et al.'s study on 171 SLE patients and 50 normal healthy control subjects, where they found that raised IL-6 levels were not correlated to disease activity index assessed by using the British Isles Lupus Assessment Group (BILAG) and other serological activity, except for an inverse correaltion with anemia $\left(\mathrm{rho}_{\mathrm{p}}\right.$ : $\left.-0.190, \mathrm{p}=0.002\right)$ (21). An earlier study by Grondal et al. on 52 SLE patients found no statistical correlation between IL-6 and SLE disease activity, which was assessed using the SLEDAI and SLAM (22). However, Abdel Galil et al. recently published a study of 72 female SLE patients and 70 healthy subjects, where they assessed SLE disease activity using SLEDAI and found higher IL-6 and IL-17 levels in SLE patients and a significantly higher level of cytokines in the active lupus nephritis subgroup (rho=0.497, $\mathrm{P}=0.005$ ) (23). The present study was unable to obtain a similar correlation, which may be attributed to the different methods of analysis and the long serum IL-6 half-life, which was reported to be up to 15 hours (24). It may also be attributed to the subgroup population of this study, whereby the researchers looked into the subset of SLE patients who had lupus nephritis. The presence of a soluble IL-6 receptor was reported in a previous study and may have contributed to the prolonged half-life of the IL- 6 and association with disease activity (25).

\section{Therapeutic benefit from alteration of serum prolactin in systemic lupus erythematosus}

In the murine sample, the treatment with prolactin inhibitor (bromocriptine) was associated with a delayed elevation of antidsDNA antibodies and serum IgG. The treatment arm had a better survival rate compared to the control group (26). A pilot study by Jara et al. randomized 20 SLE pregnant ladies during their second trimester for bromocriptine treatment in combination with prednisolone $10 \mathrm{mg}$ once daily against prednisolone treatment only. The results were remarkable, as they found similar significant reductions of serum prolactin levels at week $30(\mathrm{p}<0.02)$ and week $35(\mathrm{p}<0.002)$ of pregnancy with no flare in the treatment arm, no premature rupture of the membrane, and $80 \%$ of the pregnancies ended with birth at term (27). To date, there has been increasing evidence regarding bromocriptine safety and tolerability, including headache, vertigo, and nausea with no increment of congenital anomaly or abortions $(27,28)$. The researchers believe that a well-designed and larger randomized clinical trial in the future should be performed to evaluate the therapeutic benefits of bromocriptine in pregnant and non-pregnant SLE patients. 


\section{Clinical implication}

The findings of this study discovered a significant positive correlation between serum prolactin level with lupus nephritis disease activity assessed by SLEDAI-2000 and, on the basis of the excellent PPV of $97.1 \%$, supported the possibility that serum prolactin may be one of the important biochemical markers in the future.

\section{CONCLUSIONS}

Serum prolactin was positively correlated with SLE disease activity assessed using SLEDAI score. The additional findings in our subset of patients resulted in positive correlations between serum prolactin with the urine protein creatinine index in lupus nephritis patients.

Acknowledgement: I would like to thank the Dean of Medical Faculty UKM and Director of UKM Hospital for their cooperation to produce this article.

\section{REFERENCES}

1. Jasmin R, Sockalingam S, Cheah TE, Goh KJ. Systemic lupus erythematosus in the multiethnic Malaysian population: disease expression and ethnic differences revisited. Lupus. 2013; 22: 967-71.

2. Wang F, Wang CL, Tan CT, Manivasagar M. Systemic lupus erythematosus in Malaysia: a study of 539 patients and comparison of prevalence and disease expression in different racial and gender groups. Lupus. 1997; 6: 248-53.

3. Treadwell EL, Wiley K, Word B, et al. Prolactin and Dehydroepiandrosterone Levels in Women with Systemic Lupus Erythematosus: The Role of the Extrapituitary Prolactin Promoter Polymorphism at $-1149 \mathrm{G} / \mathrm{T}$. J Immunol Res. 2015; 2015: 435658.

4. Orbach H, Shoenfeld Y. Hyperprolactinemia and autoimmune diseases. Autoimmun Rev. 2007; 6: 537-42.

5. Bole-Feysot C, Goffin V, Edery M, et al. Prolactin (PRL) and its receptor: actions, signal transduction pathways and phenotypes observed in PRL receptor knockout mice. Endocr Rev. 1998; 19: 225-68.

6. Ben-Jonathan N, Mershon JL, Allen DL, Steinmetz RW. Extrapituitary prolactin: distribution, regulation, functions, and clinical aspects. Endocr Rev. 1996; 17: 639-69.

7. Jara LJ, Vera-Lastra O, Miranda JM, et al. Pro- lactin in human systemic lupus erythematosus. Lupus. 2001; 10: 748-56.

8. Shelly S, Boaz M, Orbach H. Prolactin and autoimmunity. Autoimmun Rev. 2012; 11: A465-70.

9. Rezaieyazdi Z, Hesamifard A. Correlation between serum prolactin levels and lupus activity. Rheumatol Int. 2006; 26: 1036-9.

10. Karimifar M, Tahmasebi A, Bonakdar ZS, Purajam S. Correlation of serum prolactin levels and disease activity in systematic lupus erythematosus. Rheumatol Int. 2013; 33: 511-6.

11. Allen SH, Sharp GC, Wang G, et al. Prolactin levels and antinuclear antibody profiles in women tested for connective tissue disease. Lupus. 1996; 5: 30-7.

12. Kelly PA, Ali S, Rozakis M, et al. The growth hormone/prolactin receptor family. Recent Progress Horm Res. 1993; 48: 123-64.

13. Dostal C, Moszkorzova L, Musilova L, et al. Serum prolactin stress values in patients with systemic lupus erythematosus. Ann Rheum Dis. 2003; 62: 487-8.

14. Jacobi AM, Rohde W, Ventz M, et al. Enhanced serum prolactin (PRL) in patients with systemic lupus erythematosus: PRL levels are related to the disease activity. Lupus. 2001; 10: 554-61.

15. Leanos-Miranda A, Cardenas-Mondragon G. Serum free prolactin concentrations in patients with systemic lupus erythematosus are associated with lupus activity. Rheumatology (Oxford, England). 2006; 45: 97-101.

16. Huang CM, Chou CT. Hyperprolactinemia in systemic lupus erythematosus. Zhonghua yi xue za zhi = Chin Med J. 1997; 59: 37-41.

17. Garcia M, Colombani-Vidal ME, Zylbersztein $\mathrm{CC}$, et al. Analysis of molecular heterogeneity of prolactin in human systemic lupus erythematosus. Lupus. 2004; 13: 575-83.

18. Ugarte-Gil MF, Gamboa-Cardenas RV, Zevallos F, et al. High prolactin levels are independently associated with damage accrual in systemic lupus erythematosus patients. Lupus. 2014; 23: 969-74.

19. Kuroiwa T, Lee EG. Cellular interactions in the pathogenesis of lupus nephritis: the role of $\mathrm{T}$ cells and macrophages in the amplification of the inflammatory process in the kidney. Lupus. 1998; 7: 597-603.

20. Cai YT, Peng XB. [Influence of serum prolactin on interleukin- 6 secretion by peripheral blood mononuclear cells in patients with systemic lupus erythematosus]. Nan fang yi ke da xue xue bao = J South Med Univ. 2011; 31: 1611-3.

21. Ripley BJ, Goncalves B, Isenberg DA, et al. Raised levels of interleukin 6 in systemic lupus erythematosus correlate with anaemia. Ann Rheum Dis. 2005; 64: 849-53. 
22. Grondal G, Gunnarsson I, Ronnelid J, et al. Cytokine production, serum levels and disease activity in systemic lupus erythematosus. Clin Exp Rheumatol. 2000; 18: 565-70.

23. Abdel Galil SM, Ezzeldin N, El-Boshy ME. The role of serum IL-17 and IL-6 as biomarkers of disease activity and predictors of remission in patients with lupus nephritis. Cytokine. 2015; 76: 280-7.

24. Wirtz DC, Heller KD, Miltner O, et al. Interleukin-6: a potential inflammatory marker after total joint replacement. Int Orthop. 2000; 24: 194-6.

25. Jones SA, Horiuchi S, Topley N, et al. The soluble interleukin 6 receptor: mechanisms of production and implications in disease. FASEB J. 2001; 15: 43-58.

26. McMurray R, Keisler D, Kanuckel K, et al. Prolactin influences autoimmune disease activity in the female $\mathrm{B} / \mathrm{W}$ mouse. J Immunol (Baltimore, Md: 1950). 1991; 147: 3780-7.

27. Jara LJ, Cruz-Cruz P, Saavedra MA, et al. Bromocriptine during pregnancy in systemic lupus erythematosus: a pilot clinical trial. Ann N Y Acad Sci. 2007; 1110: 297-304.

28. Qian Q, Liuqin L, Hao L, et al. The effects of bromocriptine on preventing postpartum flare in systemic lupus erythematosus patients from South China. J Immunol Res. 2015; 2015: 316965. 